\title{
On Yamabe constants of Riemannian products
}

\author{
Kazuo Akutagawa, Luis A. Florit and Jimmy Petean
}

For a closed Riemannian manifold $\left(M^{m}, g\right)$ of constant positive scalar curvature and any other closed Riemannian manifold $\left(N^{n}, h\right)$, we show that the limit of the Yamabe constants of the Riemannian products $(M \times N, g+r h)$ as $r$ goes to infinity is equal to the Yamabe constant of $\left(M^{m} \times \mathbb{R}^{n},\left[g+g_{\mathbb{E}}\right]\right)$ and is strictly less than the Yamabe invariant of $S^{m+n}$ provided $n \geq 2$. We then consider the minimum of the Yamabe functional restricted to functions of the second variable and we compute the limit in terms of the best constants of the Gagliardo-Nirenberg inequalities.

\section{Introduction}

Let $M^{m}$ be a closed smooth manifold of dimension $m$ and denote by $[g]$ the conformal class of a Riemannian metric $g$ on $M$. The Yamabe constant $Y(M,[g])$ of $[g]$ is the infimum of the normalized total scalar curvature functional restricted to $[g]$ :

$$
Y(M,[g])=\inf _{h \in[g]} \frac{\int_{M} \mathbf{s}_{h} d \mu_{h}}{\operatorname{Vol}(M, h)^{(m-2) / m}},
$$

where $\mathbf{s}_{h}$ is the scalar curvature of $h$ and $d \mu_{h}$ its volume element. Of course, the Yamabe constant can be expressed in terms of functions in the Sobolev space $W^{1,2}(M)$ (by writing $h=f^{4 /(m-2)} g$ if $f \in C_{+}^{\infty}(M)$ ):

$$
Y(M,[g])=\inf _{\substack{f \in W^{1,2}(M) \\ f \neq 0}} Q_{g}(f):=\inf _{\substack{f \in W^{1,2}(M) \\ f \neq 0}} \frac{\int_{M}\left(a_{m}|\nabla f|_{g}^{2}+\mathbf{s}_{g} f^{2}\right) d \mu_{g}}{\|f\|_{p_{m}}^{2}},
$$

where $a_{m}=\frac{4(m-1)}{m-2}$ and $p_{m}=\frac{2 m}{m-2}$.

Functions realizing the infimum are called Yamabe minimizers and the corresponding metrics are called Yamabe metrics (and have constant scalar curvature). They always exist by a fundamental theorem obtained in several steps by Yamabe [29], Trudinger [27], Aubin [3] and Schoen [21]. Then one defines the Yamabe invariant of $M, Y(M)$, as the supremum of the Yamabe constants of all conformal classes of metrics on $M[11,12,22]$. This 
invariant is always finite since for any conformal class $[g], Y\left(M^{m},[g]\right) \leq$ $Y\left(S^{m},\left[g_{S^{m}}\right]\right)=m(m-1) \operatorname{Vol}\left(S^{m}, g_{S^{m}}\right)^{2 / m}$, where $g_{S^{m}}$ is the round metric on $S^{m}$ of constant sectional curvature 1 . We will denote $\mathbf{Y}_{m}:=Y\left(S^{m}\right)=$ $Y\left(S^{m},\left[g_{S^{m}}\right]\right)$.

The nature of the problem of computing or estimating the Yamabe constant of a conformal class, and therefore the Yamabe invariant of the manifold, depends strongly on whether the constant is positive or non-positive. If $Y(M,[g]) \leq 0$, then $Y(M,[g]) \geq\left(\inf _{M} \mathbf{s}_{g}\right) \operatorname{Vol}(M, g)^{2 / m}$, as was first pointed out by Kobayashi in [12]. This allows, for instance, to study the behavior of the Yamabe invariant under surgery [18] and so to obtain some understanding of the invariant in the non-positive case $[6,19,20]$. Such an estimate does not exist in the positive case. In particular, there might exist unit volume Riemannian metrics on $M^{m}$ of constant scalar curvature greater than $\mathbf{Y}_{m}$. A typical example of this situation comes from Riemannian products: if $\left(M^{m}, g\right)$ and $\left(N^{n}, h\right)$ are unit volume Riemannian manifolds of constant scalar curvature and $\mathbf{s}_{g}>0$, then, for small $\delta>0, \delta^{n} g+\delta^{-m} h$ has volume one and scalar curvature greater than $\mathbf{Y}_{m+n}$. It is the main purpose of this article to study the Yamabe constants of such Riemannian products.

There is one well-understood example in this direction worked out by Kobayashi [11,12] and Schoen [22]: for any $r>0$, all solutions of the Yamabe equation on $\left(S^{n-1} \times S^{1}, g_{S^{n-1}}+r g_{S^{1}}\right)$ depend only on the $S^{1}$-variable and one can understand the solutions of the resulting ordinary differential equation. Following this lead, we will consider the Riemannian products $\delta^{n} g+\delta^{-m} h$ on $M \times N$ and look for solutions of the Yamabe equations which depend only on the second variable.

Let

$$
Y_{N}(M \times N, g+h):=\inf _{\substack{f \in W^{1,2}(N) \\ f \neq 0}} Q_{g+h}(f) .
$$

One can see that the infimum is realized and that if $f$ is such a minimizer then $f^{4 /(m+n-2)}(g+h)$ has constant scalar curvature (we will go over this on Section 2). We remark that, contrarily to the Yamabe constant $Y(M \times$ $N,[g+h])$, this constant $Y_{N}(M \times N, g+h)$ is not a conformal invariant, but merely a scale invariant.

Our first result says in particular that the limit of the Yamabe constant of the products above exists:

Theorem 1.1. Let $\left(M^{m}, g\right)$ be a closed Riemannian $m$-manifold $(m \geq 2)$ of positive scalar curvature (not necessarily constant) and $\left(N^{n}, h\right)$ any closed 
Riemannian n-manifold. Then,

$\lim _{r \nearrow \infty} Y(M \times N,[g+r h])=Y\left(M \times \mathbb{R}^{n},\left[g+g_{\mathbb{E}}\right]\right):=\inf _{\substack{f \in C_{c}^{\infty}\left(M \times \mathbb{R}^{n}\right) \\ f \neq 0}} Q_{g+g_{\mathbb{E}}}(f)>0$,

and

$\lim _{r \nearrow \infty} Y_{N}(M \times N, g+r h)=Y_{\mathbb{R}^{n}}\left(M \times \mathbb{R}^{n}, g+g_{\mathbb{E}}\right):=\inf _{\substack{f \in C_{c}^{\infty}\left(\mathbb{R}^{n}\right) \\ f \neq 0}} Q_{g+g_{\mathbb{E}}}(f)>0$,

where $g_{\mathbb{E}}$ stands for the Euclidean metric on $\mathbb{R}^{n}$.

Remark 1.2. The fact that $Y\left(S^{n-1} \times S^{1}\right)=Y\left(S^{n}\right)$ (for $n \geq 3$ ) was first proved by Kobayashi [11] and Schoen [22], by analysis of the behavior of constant scalar curvature metrics on $\left(\mathbb{R}^{n}-\{0\}, g_{\mathbb{E}}\right)$. Another proof was given by also Kobayashi [12], by using an argument in the proof of the celebrated Kobayashi's inequality [12, Theorem 2]. The above theorem gives the third proof since

$$
Y\left(S^{n-1} \times S^{1}\right) \geq Y\left(S^{n-1} \times \mathbb{R}^{1},\left[g_{S^{n-1}}+g_{\mathbb{E}}\right]\right)=Y\left(S^{n},\left[g_{S^{n}}\right]\right)=Y\left(S^{n}\right)
$$

On the constant $Y\left(M \times \mathbb{R}^{n},\left[g+g_{\mathbb{E}}\right]\right)$, we also obtain the following theorem.

Theorem 1.3. Let $\left(M^{m}, g\right)$ be a closed Riemannian m-manifold $(m \geq 2)$ of positive scalar curvature (not necessarily constant). Assume that $n \geq 2$. Then,

$$
Y\left(M \times \mathbb{R}^{n},\left[g+g_{\mathbb{E}}\right]\right)<\mathbf{Y}_{m+n} .
$$

Recall that the best $n$-dimensional Sobolev constant is the smallest positive number $\sigma_{n}$ such that for any smooth compactly supported function $f$ on $\mathbb{R}^{n},\|f\|_{p_{n}}^{2} \leq \sigma_{n}\|\nabla f\|_{2}^{2}$. Due to the conformal invariance of the Yamabe constant, one can use the stereographic projection to translate the Yamabe functional from the round sphere to the Euclidean space to obtain:

$$
\sigma_{n}=\frac{a_{n}}{\mathbf{Y}_{n}}
$$

In a similar fashion, we will see that when studying the limits above a fundamental role is played by the best constant in the Gagliardo-Nirenberg 
inequalities: namely, we will call $\sigma_{m, n}$ the smallest positive number such that for any $f \in W^{1,2}\left(\mathbb{R}^{n}\right)$,

$$
\|f\|_{p_{m+n}}^{2} \leq \sigma_{m, n}\|\nabla f\|_{2}^{2 n /(m+n)}\|f\|_{2}^{2 m /(m+n)} .
$$

Or what is equivalent:

$$
\sigma_{m, n}=\left(\inf _{\substack{f \in W^{1,2}\left(\mathbb{R}^{n}\right) \\ f \neq 0}} \frac{\|\nabla f\|_{2}^{2 n /(m+n)}\|f\|_{2}^{2 m /(m+n)}}{\|f\|_{p_{m+n}}^{2}}\right)^{-1} .
$$

The constant $\sigma_{m, n}$ is of classical interest in the study of partial differential equations and has been computed numerically, although it is not known any explicit expression for the constant or for the minimizing function (which is known to be radial and decreasing) [8,9,13,16,17,28].

We will prove the following.

Theorem 1.4. Let $\left(M^{m}, g\right)$ be a closed smooth unit volume Riemannian manifold of constant positive scalar curvature $\mathbf{s}_{g}$. Then

$$
Y_{\mathbb{R}^{n}}\left(M \times \mathbb{R}^{n}, g+g_{\mathbb{E}}\right)=\frac{C(m, n) \mathbf{s}_{g}^{m /(m+n)}}{\sigma_{m, n}},
$$

where $C(m, n)=\left(a_{m+n}\right)^{n /(m+n)}(m+n) n^{-n /(m+n)} m^{-m /(m+n)}$.

It is clear that

$$
Y\left(M \times \mathbb{R}^{n},\left[g+g_{\mathbb{E}}\right]\right) \leq Y_{\mathbb{R}^{n}}\left(M \times \mathbb{R}^{n}, g+g_{\mathbb{E}}\right),
$$

and it seems that the equality should hold under certain hypothesis. It certainly cannot always be the case since $Y_{\mathbb{R}^{n}}\left(M \times \mathbb{R}^{n}, g+g_{\mathbb{E}}\right)>\mathbf{Y}_{m+n}$ if $\mathbf{s}_{g}$ is big enough.

It is then natural to ask the following. Question: Is it true that $Y_{\mathbb{R}^{n}}\left(M \times \mathbb{R}^{n}, g+g_{\mathbb{E}}\right)=Y\left(M \times \mathbb{R}^{n},\left[g+g_{\mathbb{\mathbb { B }}}\right]\right)$ if $g$ is a Yamabe metric?

As we mentioned before, the constants $\sigma_{m, n}$ can be explicitly computed numerically. Using these computations, we apply Theorem 1.4 to the case when $(M, g)$ and $(N, h)$ are round spheres. These are particularly interesting cases because of Schoen and Kobayashi's argument mentioned above and because $S^{n} \times S^{m}$ is obtained by performing surgery on $S^{m+n}$, and therefore 
if the surgery theorem in [18] were true in the positive case we should have $Y\left(S^{m} \times S^{n}\right)=\mathbf{Y}_{m+n}$. Set

$$
\begin{aligned}
Y_{S^{n}}^{\infty}\left(S^{m} \times S^{n}\right) & :=\lim _{r \nearrow \infty} Y_{S^{n}}\left(S^{m} \times S^{n}, g_{S^{m}}+r g_{S^{n}}\right) \\
& =Y_{\mathbb{R}^{n}}\left(S^{m} \times \mathbb{R}^{n}, g_{S^{m}}+g_{\mathbb{E}}\right) .
\end{aligned}
$$

We give the corresponding values for all $m, n \geq 2$ with $m+n \leq 9$.

\begin{tabular}{ccccc}
\hline$m$ & $n$ & $\sigma_{m, n}^{-1}$ & $Y_{S^{n}}^{\infty}\left(S^{m} \times S^{n}\right)$ & $\mathbf{Y}_{m+n}$ \\
\hline 2 & 2 & 2.41877 & 59.40481 & 61.56239 \\
2 & 3 & 3.87947 & 75.39687 & 78.99686 \\
3 & 2 & 2.11360 & 78.18644 & 78.99686 \\
2 & 4 & 5.66408 & 91.68339 & 96.29728 \\
3 & 3 & 3.19925 & 94.71444 & 96.29728 \\
4 & 2 & 1.90282 & 95.87367 & 96.29728 \\
2 & 5 & 7.71937 & 108.1625 & 113.5272 \\
3 & 4 & 4.53960 & 111.2934 & 113.5272 \\
4 & 3 & 2.75810 & 112.6214 & 113.5272 \\
5 & 2 & 1.75469 & 113.2670 & 113.5272 \\
2 & 6 & 10.0021 & 124.7747 & 130.7157 \\
3 & 5 & 6.10843 & 127.9414 & 130.7157 \\
4 & 4 & 3.81586 & 129.3551 & 130.7157 \\
5 & 3 & 2.45567 & 130.1272 & 130.7157 \\
6 & 2 & 1.64650 & 130.5398 & 130.7157 \\
2 & 7 & 12.4764 & 141.4740 & 147.8778 \\
3 & 6 & 7.88171 & 144.6521 & 147.8778 \\
4 & 5 & 5.06274 & 146.1089 & 147.8778 \\
5 & 4 & 3.32083 & 146.9519 & 147.8778 \\
6 & 3 & 2.23778 & 147.4615 & 147.8778 \\
7 & 2 & 1.56455 & 147.7507 & 147.8778 \\
\hline & & & &
\end{tabular}

It should be the case that $Y_{S^{n}}^{\infty}\left(S^{m} \times S^{n}\right)<\mathbf{Y}_{n+m}$ for all values $m, n \geq 2$. Hence, this gives a proof of Theorem 1.3 for $\left(M^{m}, g\right)=\left(S^{m}, g_{S^{m}}\right)$, namely that of

$$
Y\left(S^{m} \times \mathbb{R}^{n},\left[g_{S^{m}}+g_{\mathbb{E}}\right]\right)<\mathbf{Y}_{m+n},
$$


when $m, n \geq 2, m+n \leq 9$. Moreover, the above method gives a numerical estimate from above for the constant $Y\left(S^{m} \times \mathbb{R}^{n},\left[g_{S^{m}}+g_{\mathbb{E}}\right]\right)$.

Note also that in the 4-dimensional case the Yamabe invariant of $\mathbb{C P}^{2}$ is realized by the conformal class of the Fubini-Study metric $g_{\mathrm{FS}}[10,14]$, giving

$$
Y\left(\mathbb{C P}^{2}\right)=Y\left(\mathbb{C P},\left[g_{\mathrm{FS}}\right]\right)=12 \sqrt{2} \pi=53.31459 \ldots
$$

and since Einstein metrics are always Yamabe metrics we have that

$$
Y\left(S^{2} \times S^{2},\left[g_{S^{2}}+g_{S^{2}}\right]\right)=16 \pi=50.26548 \ldots
$$

In the next section, we will review some known results on Yamabe constants, point out a few observations and fix some notation. In Section 3, we will recall Schoen and Kobayashi's discussion of the solutions of the Yamabe equation on $\left(S^{n-1} \times S^{1}, g_{S^{n-1}}+r g_{S^{1}}\right)$. We will prove Theorems 1.1 and 1.3 in Section 4 and Theorem 1.4 in Section 5. Finally, a procedure to compute numerically these Yamabe constants is given in the last section.

\section{Preliminaries}

Let $\left(X^{k}, g\right)$ be a closed smooth $k$-dimensional Riemannian manifold. Recall that $\mathbf{s}_{g}$ is the scalar curvature of $g, d \mu_{g}$ its volume element and

$$
p=p_{k}=\frac{2 k}{k-2} \quad \text { and } \quad a=a_{k}=\frac{4(k-1)}{k-2} .
$$

Consider the Sobolev space $W^{1,2}(X)$ and the Yamabe functional defined by

$$
f \longmapsto Q_{g}(f):=\frac{\int_{X}\left(a_{k}|\nabla f|_{g}^{2} d \mu_{g}+\mathbf{s}_{g} f^{2}\right) d \mu_{g}}{\|f\|_{p}^{2}} .
$$

We say that $f$ is a Yamabe minimizer (for $g$ ) if it realizes the minimum of the Yamabe functional. In this case, $f^{4 /(k-2)} g$ has a constant scalar curvature and the Yamabe constant of the conformal class of $g$ is then equal to $Q_{g}(f)$.

In this paper, we consider a unit volume Riemannian product $\left(M^{m} \times\right.$ $\left.N^{n}, g+h\right)$. We assume that the scalar curvature of both $g$ and $h$ is constant and try to understand the Yamabe constant of the conformal class of the product metric. This is of no interest if $\mathbf{s}_{g}+\mathbf{s}_{h}$ is negative, since in this case we have uniqueness of the Yamabe metric. The situation we want to address is when $\mathbf{s}_{g}+\mathbf{s}_{h}$ is positive and bigger than $\mathbf{Y}_{m+n}$, the Yamabe invariant of the round sphere $S^{m+n}$. In this case, there must exist a non-constant 
Yamabe function, and so another metric of constant scalar curvature in the same conformal class.

To compute the Yamabe constant is a very difficult problem and so it is to understand the Yamabe minimizer. We will then restrict ourselves to functions which depend only on one of the variables, that is, positive smooth functions $f: N^{n} \rightarrow \mathbb{R}$ of one of the factors in the Riemannian product $M^{m} \times$ $N^{n}$. The scalar curvature of $f^{4 /(m+n-2)}(g+h)$ is given by

$$
\mathbf{s}_{g+h}=f^{1-p_{m+n}}\left(-a_{m+n} \Delta_{h} f+\mathbf{s}_{g+h} f\right) .
$$

We then introduce the following definition.

Definition 2.1. Given a Riemannian product $(M \times N, g+h)$ of constant scalar curvature manifolds, the $N$-Yamabe constant of $(M \times N, g+h)$ is the infimum of the $(g+h)$-Yamabe functional restricted to $W^{1,2}(N)$. We will denote this constant by $Y_{N}(M \times N, g+h)$.

To study critical points of the $(g+h)$-Yamabe functional restricted to $W^{1,2}(N)$, let $\varphi, f: N \rightarrow \mathbb{R}$ be smooth functions. A well-known computation gives that

$$
\begin{aligned}
\frac{d(Q(f+t \varphi))}{d t}(0)= & \frac{2 \operatorname{Vol}(M, g)}{\|f\|_{p}^{2}} \\
& \times \int_{N}\left(-a_{m+n} \Delta_{h} f+\mathbf{s}_{g+h} f-\|f\|_{p}^{-p+2} Q(f) f^{p-1}\right) \varphi d \mu_{h},
\end{aligned}
$$

where $p=p_{m+n}$ and $Q(\cdot)=Q_{g+h}(\cdot)$. Therefore, the critical points of the Yamabe functional restricted to $W^{1,2}(N)$ are precisely the functions $f$ such that the conformal metric $f^{4 /(m+n-2)}(g+h)$ has constant scalar curvature $\tilde{\mathbf{s}}=\|f\|_{p}^{-p+2} Q(f)$. The next point is that the infimum of the Yamabe functional restricted to $W^{1,2}(N)$ is always achieved. This is a simple fact; it is essentially the subcritical case of the Yamabe problem, but we sketch its proof since we have not seen it in the literature.

Proposition 2.2. Let $\left(M^{m}, g\right)$ and $\left(N^{n}, h\right)$ be closed Riemannian manifolds of constant scalar curvature. Then, there exists a positive smooth function $f$ on $N^{n}$ which minimizes the Yamabe quotient among all functions on $N^{n}$.

Proof. Without loss of generality, we may assume that $\operatorname{Vol}(M, g)=1$. Let $\left\{u_{i}\right\}$ be a sequence of non-negative functions on $N$ such that $Q_{g+h}\left(u_{i}\right) \rightarrow$ 
$Y_{N}(M \times N, g+h)$. We can assume that $\left\|u_{i}\right\|_{p_{k}}=1$, where $k=m+n$. Then,

$$
\begin{aligned}
\left\|u_{i}\right\|_{2,1}^{2} & =\int_{N}\left(\left|\nabla u_{i}\right|_{h}^{2}+u_{i}^{2}\right) d \mu_{h} \\
& =\frac{1}{a_{k}} Q_{g+h}\left(u_{i}\right)+\int_{N}\left(1-\frac{\mathbf{s}_{g+h}}{a_{k}}\right) u_{i}^{2} d \mu_{h} \\
& \leq \frac{Y_{N}(M \times N, g+h)+1}{a_{k}}+K\left\|u_{i}\right\|_{2}^{2},
\end{aligned}
$$

for some $K>0$, that is bounded independently of $i$ since $\left\|u_{i}\right\|_{2}^{2} \leq\left\|u_{i}\right\|_{p_{k}}^{2}$ $\operatorname{Vol}(N, h)^{2 / k}=\operatorname{Vol}(N, h)^{2 / k}$ by Hölder's inequality. Since

$$
\frac{1}{p_{k}}>\frac{1}{2}-\frac{1}{n}
$$

by the Rellich-Kondrakov theorem, the inclusion $W^{1,2}(N) \subset L^{p_{k}}(N)$ is a compact operator. Therefore, there exists a subsequence of the $\left\{u_{i}\right\}$ which converges weakly in $W^{1,2}(N)$ and strongly in $L^{p_{k}}(N)$ to a function $u \in$ $W^{1,2}(N)$ with $\|u\|_{p_{k}}=1$.

Now, by the weak convergence in $W^{1,2}(N)$, we have

$$
\||\nabla u|\|_{2}^{2}=\lim _{i \rightarrow \infty} \int_{N}\left\langle\nabla u, \nabla u_{i}\right\rangle d \mu_{h},
$$

and therefore

$$
\left\|\left|\nabla u\left\|\left.\right|_{2} ^{2} \leq \limsup _{i \rightarrow \infty}\right\|\right| \nabla u_{i}\right\|_{2}^{2} .
$$

And since by strong convergence in $L^{2}$

$$
\int_{N} \mathbf{s}_{g+h} u^{2} d \mu_{h}=\lim _{i \rightarrow \infty} \int_{N} \mathbf{s}_{g+h} u_{i}^{2} d \mu_{h}
$$

we have that $Q_{g+h}(u) \leq \lim _{i \rightarrow \infty} Q_{g+h}\left(u_{i}\right)$, and hence $Q_{g+h}(u)=Y_{N}(M \times$ $N, g+h)$. It then follows from elliptic regularity that $u$ has to be strictly positive and smooth.

Remark 2.3. Note that, for a given Riemannian product of constant scalar curvature, we have three associated Yamabe constants each producing a constant scalar curvature metric. The three are equal if the original product is a Yamabe metric. 


\section{Reviewing the circle}

Kobayashi [11] and Schoen [22] gave a fairly complete study of the solutions of the Yamabe equation for the manifolds $\left(S^{n-1} \times S^{1}, g_{S^{n-1}}+r g_{S^{1}}\right)$, where $n \geq 2$ and $r$ is a positive constant. For instance, Schoen points out that due to the conformal invariance and a theorem of Caffarelli-Gidas-Spruck [7], all solutions are functions of $S^{1}$. Moreover, he writes down the Yamabe equation for a function of $S^{1}$. Moving to the Riemannian universal covering $\left(S^{n-1} \times \mathbb{R}, g_{S^{n-1}}+d t^{2}\right)$, one has to deal with the ordinary differential equation:

$$
\frac{d^{2} u}{d t^{2}}-\frac{1}{4}(n-2)^{2} u+\frac{n(n-2)}{4} u^{(n+2) /(n-2)}=0,
$$

and look for solutions which are $2 \pi r$-periodic. Note that exactly the same equation shows up if we consider a Riemannian product $\left(M \times S^{1}, g+r g_{S^{1}}\right)$, where $M$ is $(n-1)$-dimensional and the scalar curvature of $g$ is $(n-1)(n-$ $2)$. In this way, one can understand all constant scalar curvature metrics which are conformal by a function of $S^{1}$ to $\left(M \times S^{1}, g+r g_{S^{1}}\right)$; the solutions are the same as those for $S^{n-1}$ discussed in [11,22]. So for $r$ close to 1 , there is going to be only one solution, and as $r$ increases, the number of solutions will increase. If $\operatorname{Vol}(M, g)=V_{n-1}:=\operatorname{Vol}\left(S^{n-1}, g_{S^{n-1}}\right)$, then the $S^{1}$-Yamabe constant of the product will be less than $\mathbf{Y}_{n}$ for all $r$ and will approach $\mathbf{Y}_{n}$ as $r$ goes to infinity.

Example 3.1. If $\operatorname{Vol}(M, g)>V_{n-1}$ in the discussion above, the number of solutions will still be the same, but as $r$ becomes big the $S^{1}$-Yamabe constants of the product will be bigger than $\mathbf{Y}_{n}$. In particular, the $S^{1}$ Yamabe constant will be greater than the Yamabe constant.

\section{Proofs of Theorems 1.1 and 1.3}

Proof of Theorem 1.1. To simplify the notation, we set $g_{r}:=g+r h$ on $M^{m} \times N^{n}$ and $\bar{g}:=g+g_{\mathbb{E}}$ on $M^{m} \times \mathbb{R}^{n}$. We may also assume that $\operatorname{Vol}(M, g)=1$.

First, we show the following:

$$
Y\left(M \times \mathbb{R}^{n},[\bar{g}]\right)>0 .
$$

Note that $\left(M \times \mathbb{R}^{n}, \bar{g}\right)$ is a complete Riemannian manifold with strictly positive injective radius and bounded sectional curvature. Under these conditions, the Sobolev embedding $W^{1,2}\left(M \times \mathbb{R}^{n}\right) \hookrightarrow L^{p}\left(M \times \mathbb{R}^{n}\right)$ holds 
(cf. [4, Theorem 2.21]), that is, there exists constant $K_{1}>0$ such that

$$
\|f\|_{p}^{2} \leq K_{1}\|f\|_{2,1}^{2} \text { for } f \in W^{1,2}\left(M \times \mathbb{R}^{n}\right)
$$

where $p=p_{m+n}:=\frac{2(m+n)}{m+n-2}$. This and the positivity of the scalar curvature of $(M, g)$ imply that

$$
\left(\int_{M \times \mathbb{R}^{n}}|f|^{p} d \mu_{\bar{g}}\right)^{2 / p} \leq \frac{K_{1}}{\alpha} \int_{M \times \mathbb{R}^{n}}\left(a_{m+n}|\nabla f|_{\bar{g}}^{2}+\mathbf{s}_{g} f^{2}\right) d \mu_{\bar{g}}
$$

for $f \in W^{1,2}\left(M \times \mathbb{R}^{n}\right)$, where $\alpha:=\min \left\{a_{m+n}, \min _{M} \mathbf{s}_{g}\right\}>0$, and hence

$$
Y\left(M \times \mathbb{R}^{n},[\bar{g}]\right) \geq \frac{\alpha}{K_{1}}>0
$$

We also have

$$
Y_{\mathbb{R}^{n}}\left(M \times \mathbb{R}^{n}, \bar{g}\right) \geq Y\left(M \times \mathbb{R}^{n},[\bar{g}]\right)>0 .
$$

Second, we prove the following

$$
\liminf _{r \nearrow \infty} Y\left(M \times N,\left[g_{r}\right]\right) \geq Y\left(M \times \mathbb{R}^{n},[\bar{g}]\right)
$$

and

$$
\liminf _{r \nearrow \infty} Y_{N}\left(M \times N, g_{r}\right) \geq Y_{\mathbb{R}^{n}}\left(M \times \mathbb{R}^{n}, \bar{g}\right)
$$

Pick any $\varepsilon>0$. There exist a small constant $\delta>0$ and finite points $\left\{q_{1}, \ldots, q_{\ell}\right\} \subset N$ such that

- $\left\{U_{k}:=\exp _{q_{k}}^{h}\left(\mathbf{B}_{\delta}^{h}(0)\right)\right\}_{k=1}^{\ell}$ is an open covering of $\mathrm{N}$ and that, on each $U_{k}$ with respect to $h$-normal coordinates $x=\left(x^{1}, \ldots\right.$, $\left.x^{n}\right)$ at $q_{k}$,

- $(1+\varepsilon)^{-1} \delta_{i j} d x^{i} d x^{j} \leq h_{i j} d x^{i} d x^{j} \leq(1+\varepsilon) \delta_{i j} d x^{i} d x^{j}$,

- $(1+\varepsilon)^{-1} d x \leq d \mu_{h} \leq(1+\varepsilon) d x$.

Here, $\exp _{q_{k}}^{h}: \mathbf{B}_{\delta}^{h}(0):=\left\{\left.v \in T_{q_{k}} N|| v\right|_{h}<\delta\right\} \rightarrow U_{k}$ and $d x:=d x^{1} \wedge \cdots \wedge$ $d x^{n}$ denote, respectively, the $h$-exponential map at $q_{k}$ and the Euclidean volume form. Then note that, for any $r>1$, on each $U_{i}$ with respect to 
$\left(r^{2} h\right)$-normal coordinates $y=\left(y^{1}, \ldots, y^{n}\right)$ at $q_{k}$,

$$
\begin{gathered}
(1+\varepsilon)^{-1} \delta_{i j} d y^{i} d y^{j} \leq\left(r^{2} h\right)_{i j} d y^{i} d y^{j} \leq(1+\varepsilon) \delta_{i j} d y^{i} d y^{j} \\
(1+\varepsilon)^{-1} d y \leq d \mu_{r^{2} h} \leq(1+\varepsilon) d y
\end{gathered}
$$

We also note that there exists a constant $K_{2}>0$ such that

$$
\operatorname{diam}\left(U_{k}, r^{2} h\right) \geq K_{2} r \quad(k=1, \ldots, \ell)
$$

for any $r \geq 1$. Let $\left\{\eta_{k}=\chi_{k}^{2}\right\}_{k=1}^{\ell}$ be a partition of unity subordinate to the covering $\left\{U_{k}\right\}_{k=1}^{\ell}$ and $K_{3}>0$, a positive constant independent of $r \geq 1$ such that

$$
\left|\nabla \chi_{k}\right|_{h} \leq K_{3} \quad(k=1, \ldots, \ell),
$$

and hence

$$
\left|\nabla \chi_{k}\right|_{r^{2} h} \leq \frac{K_{3}}{r} \quad(k=1, \ldots, \ell) .
$$

With the above understandings, for any $r>1$ and $\varphi \in C^{\infty}(M \times N)$, we estimate the $L^{p}$-norm of $\varphi$ with respect to $g_{r}$ as follows:

$$
\begin{aligned}
\|\varphi\|_{p}^{2} & =\left\|\varphi^{2}\right\|_{p / 2}=\left\|\Sigma_{k} \chi_{k}^{2} \varphi^{2}\right\|_{p / 2} \\
& \left.\leq \Sigma_{k}\left(\int_{M \times U_{k}}\left|\chi_{k} \varphi\right|^{p} d \mu_{g_{r}}\right)\right)^{2 / p} \\
& \leq(1+\varepsilon)^{2 / p} \Sigma_{k}\left(\int_{M \times U_{k}}\left|\chi_{k} \varphi\right|^{p} d \mu_{\bar{g}}\right)^{2 / p} .
\end{aligned}
$$

Here, we identify $U_{k}=\exp _{q_{k}}^{r^{2} h}\left(\mathbf{B}_{r \delta}^{r^{2} h}(0)\right)(\subset N)$ with $\mathbb{B}_{r \delta}(0):=\left\{y \in \mathbb{R}^{n}\right.$ $|y|<r \delta\}$ via the composition of the inverse $\left(\exp _{q_{k}}^{r^{2} h}\right)^{-1}$ and the identification $\mathbf{B}_{r \delta}^{r^{2} h}(0) \cong \mathbb{B}_{r \delta}(0)$.

Set $Y_{0}:=Y\left(M \times \mathbb{R}^{n},[\bar{g}]\right)$ and $Y_{0}^{0}:=Y_{\mathbb{R}^{n}}\left(M \times \mathbb{R}^{n}, \bar{g}\right)$. We also note that, on each $M \times U_{k}\left(\subset M \times \mathbb{R}^{n}\right)$,

$$
\begin{aligned}
& \left(\int_{M \times U_{k}}\left|\chi_{k} \varphi\right|^{p} d \mu_{\bar{g}}\right)^{2 / p} \\
& \quad \leq \frac{1}{Y_{0}}\left(a_{m+n} \int_{M \times U_{k}}\left|\nabla\left(\chi_{k} \varphi\right)\right|_{\bar{g}}^{2} d \mu_{\bar{g}}+\int_{M \times U_{k}} \mathbf{s}_{g} \chi_{\ell}^{2} \varphi^{2} d \mu_{\bar{g}}\right)
\end{aligned}
$$




$$
\begin{aligned}
\leq & \frac{(1+\varepsilon)^{2}}{Y_{0}}\left(a_{m+n} \int_{M \times U_{k}}\left|\nabla\left(\chi_{k} \varphi\right)\right|_{g_{r}}^{2} d \mu_{g_{r}}+\int_{M \times U_{k}} \mathbf{s}_{g} \chi_{k}^{2} \varphi^{2} d \mu_{g_{r}}\right) \\
\leq & \frac{(1+\varepsilon)^{3}}{Y_{0}}\left(a_{m+n} \int_{M \times U_{k}} \chi_{k}^{2}|\nabla \varphi|_{g_{r}}^{2} d \mu_{g_{r}}+\int_{M \times U_{k}} \mathbf{s}_{g} \chi_{k}^{2} \varphi^{2} d \mu_{g_{r}}\right. \\
& \left.+\frac{a_{m+n} K_{3}^{2}\left(1+\varepsilon^{-1}\right)}{r^{2}} \int_{M \times U_{k}} \varphi^{2} d \mu_{g_{r}}\right) .
\end{aligned}
$$

Here, it is important to note for the proof of (4.1) that if $\varphi \in C^{\infty}(N)$, then we can replace $Y_{0}$ by $Y_{0}^{0}$.

Hence, we have

$$
\begin{aligned}
\|\mid \varphi\|_{p}^{2} \leq & \frac{(1+\varepsilon)^{3+(2 / p)}}{Y_{0}}\left(\int_{M \times N}\left(a_{m+n}|\nabla \varphi|_{g_{r}}^{2}+\mathbf{s}_{g} \varphi^{2}\right) d \mu_{g_{r}}\right. \\
& \left.+\frac{\ell a_{m+n} K_{3}^{2}\left(1+\varepsilon^{-1}\right)}{r^{2}} \int_{M \times N} \varphi^{2} d \mu_{g_{r}}\right) .
\end{aligned}
$$

From the positivity of the scalar curvature $\mathbf{s}_{g}$, there exists a large constant $r_{0}=r_{0}\left(\varepsilon, \min _{M} \mathbf{s}_{g},(N, h), m+n\right)>1$ such that

$$
\frac{\ell a_{m+n} K_{3}^{2}\left(1+\varepsilon^{-1}\right)}{r_{0}^{2}} \leq\left(\min _{M} \mathbf{s}_{g}\right) \varepsilon
$$

Therefore, we obtain

$$
\|\varphi\|_{p}^{2} \leq \frac{(1+\varepsilon)^{4+(2 / p)}}{Y_{0}} \int_{M \times N}\left(a_{m+n}|\nabla \varphi|_{g_{r}}^{2}+\mathbf{s}_{g} \varphi^{2}\right) d \mu_{g_{r}}
$$

for any $r \geq r_{0}$ and $\varphi \in C^{\infty}(M \times N)$. Again, if $\varphi \in C^{\infty}(N)$, we can replace $Y_{0}$ by $Y_{0}^{0}$. Then, this implies that

$$
Y\left(M \times N,\left[g_{r}\right]\right) \geq \frac{Y_{0}}{(1+\varepsilon)^{4+(2 / p)}}, \quad Y_{N}\left(M \times N, g_{r}\right) \geq \frac{Y_{0}^{0}}{(1+\varepsilon)^{4+(2 / p)}}
$$

for any $r \geq r_{0}$. And since $\varepsilon>0$ is arbitrary,

$$
\liminf _{r \nearrow \infty} Y\left(M \times N,\left[g_{r}\right]\right) \geq Y_{0}
$$

and

$$
\liminf _{r \nearrow \infty} Y_{N}\left(M \times N, g_{r}\right) \geq Y_{0}^{0}
$$


Finally, we prove

$$
\limsup _{r \nearrow \infty} Y\left(M \times N,\left[g_{r}\right]\right) \leq Y_{0}
$$

and

$$
\limsup _{r \nearrow \infty} Y_{N}\left(M \times N, g_{r}\right) \leq Y_{0}^{0} .
$$

Note that

$$
\lim _{\rho \nearrow \infty} Y\left(M \times \mathbb{B}_{\rho}(0),[\bar{g}]\right)=Y_{0},
$$

and

$$
\lim _{\rho \nearrow \infty} Y_{\mathbb{B}_{\rho}(0)}\left(M \times \mathbb{B}_{\rho}(0),[\bar{g}]\right)=Y_{0}^{0} .
$$

Take any small $\varepsilon>0$ and large $\rho>0$. Fix a point $q \in N$ and set an $h$-normal open neighborhood $U:=\exp _{q}^{h}\left(\mathbf{B}_{\delta}^{h}(0)\right)$ of $q$ for $\delta>0$. Here, we choose $\delta=\delta(\varepsilon,(N, h))>0$ sufficiently small satisfying the same conditions on $U$ as those in the preceding argument. Let $r_{1}>0$ be a positive constant such that $r_{1} \delta \geq \rho$. For each $r \geq r_{1}$, we also use the $\left(r^{2} h\right)$-normal coordinates $y=\left(y^{1}, \ldots, y^{n}\right)$ at $q$ on $U$ and the identification

$$
\mathbb{B}_{r \delta}(0)\left(\subset \mathbb{R}^{n}\right) \stackrel{\cong}{\longrightarrow} U=\exp _{q}^{r^{2} h}\left(\mathbf{B}_{r \delta}^{r^{2} h}(0)\right)(\subset N)
$$

With the above understandings, for any $r \geq r_{1}$ and $f \in C_{c}^{\infty}\left(M \times \mathbb{B}_{\rho}(0)\right)$ $\left(\subset C_{c}^{\infty}\left(M \times \mathbb{B}_{r \delta}(0)\right) \cong C_{c}^{\infty}(M \times U)\right)$, we obtain

$$
\begin{aligned}
\|f\|_{p}^{2} & =\left(\int_{M \times \mathbb{B}_{\rho}(0)}|f|^{p} d \mu_{\bar{g}}\right)^{2 / p} \leq(1+\varepsilon)^{2 / p}\left(\int_{M \times U}|f|^{p} d \mu_{g_{r}}\right)^{2 / p} \\
& \leq \frac{(1+\varepsilon)^{2 / p}}{Y\left(M \times N,\left[g_{r}\right]\right)} \int_{M \times U}\left(a_{m+n}|\nabla f|_{g_{r}}^{2}+\mathbf{s}_{h_{r}} f^{2}\right) d \mu_{g_{r}} \\
& \leq \frac{(1+\varepsilon)^{2+(2 / p)}}{Y\left(M \times N,\left[g_{r}\right]\right)} \int_{M \times \mathbb{B}_{\rho}(0)}\left(a_{m+n}|\nabla f|_{\bar{g}}^{2}+\left(\mathbf{s}_{g}+\frac{K_{4}}{r}\right) f^{2}\right) d \mu_{\bar{g}},
\end{aligned}
$$

where $K_{4}>0$ is a constant independent of $r$. Here, we also use the fact that $Y\left(M \times N,\left[g_{r}\right]\right)>0$ for any large $r>0$. 
In order to prove (4.2), it is important to note that for $f \in C_{c}^{\infty}\left(\mathbb{B}_{\rho}(0)\right)$, we obtain:

$$
\|f\|_{p}^{2} \leq \frac{(1+\varepsilon)^{2+(2 / p)}}{Y_{N}\left(M \times N, g_{r}\right)} \int_{M \times \mathbb{B}_{\rho}(0)}\left(a_{m+n}|\nabla f|_{\bar{g}}^{2}+\left(\mathbf{s}_{g}+\frac{K_{4}}{r}\right) f^{2}\right) d \mu_{\bar{g}} .
$$

From the positivity of $\mathbf{s}_{g}$, there exists $r_{2}=r_{2}\left(\varepsilon, \min _{M} \mathbf{s}_{g}\right)>0$ such that

$$
\mathbf{s}_{g}+\frac{K_{4}}{r} \leq(1+\varepsilon) \mathbf{s}_{g} \quad \text { on } \quad M
$$

for any $r \geq r_{2}$. Hence, for any $r \geq \max \left\{r_{1}, r_{2}\right\}$ and $f \in C_{c}^{\infty}\left(M \times \mathbb{B}_{\rho}(0)\right)$, we have

$$
\|f\|_{p}^{2} \leq \frac{(1+\varepsilon)^{3+(2 / p)}}{Y\left(M \times N,\left[g_{r}\right]\right)} \int_{M \times \mathbb{B}_{\rho}(0)}\left(a_{m+n}|\nabla f|_{\bar{g}}^{2}+\mathbf{s}_{g} f^{2}\right) d \mu_{\bar{g}} .
$$

Therefore,

$$
Y\left(M \times N,\left[g_{r}\right]\right) \leq(1+\varepsilon)^{3+(2 / p)} Y\left(M \times \mathbb{B}_{\rho}(0),[\bar{g}]\right) .
$$

Letting $r \nearrow \infty$, we then obtain

$$
\limsup _{r \nearrow \infty} Y\left(M \times N,\left[g_{r}\right]\right) \leq(1+\varepsilon)^{3+(2 / p)} Y\left(M \times \mathbb{B}_{\rho}(0),[\bar{g}]\right) .
$$

Letting also $\rho \nearrow \infty$ and $\varepsilon \searrow 0$,

$$
\limsup _{r \nearrow \infty} Y\left(M \times N,\left[g_{r}\right]\right) \leq Y_{0} .
$$

And following the same steps, we also prove (4.2). This completes the proof of Theorem 1.1.

We shall prove Theorem 1.3 by a series of lemmas. Throughout the rest of this section, we always assume the same conditions as in Theorem 1.3, that is, $m, n \geq 2$ and $\mathbf{s}_{g}>0$ on $M^{m}$. To simplify the notation, we set $\bar{g}:=g+g_{\mathbb{B}}$ on $M^{m} \times \mathbb{R}^{n}$.

By the positivity of the scalar curvature $\mathbf{s}_{g}>0$ of $g$ and the condition that $n \geq 2$, one can obtain the following.

Lemma 4.1. $\left(M^{m} \times \mathbb{R}^{n}, \bar{g}\right)$ is not locally conformally flat. Moreover, for any open set $U$ of $M^{m} \times \mathbb{R}^{n}$, the Weyl curvature $W_{\bar{g}}$ of $\bar{g}$ never vanishes identically on $U$. 
When $m+n \geq 6$, similar to Aubin's argument in [3,15, Theorem $\mathrm{B}]$, Lemma 4.1 implies the existence of a family of nice test functions $\left\{\psi_{\varepsilon}\right\}_{\varepsilon>0}$ with $Q_{\bar{g}}\left(\psi_{\varepsilon}\right)<\mathbf{Y}_{m+n}$ for sufficiently small $\varepsilon>0$. Then we obtain the following (see the proof of [2, Proposition 6.6] for details).

Lemma 4.2. Assume that $m+n \geq 6$. Then, the assertion of Theorem 1.3 holds.

Now we consider the remaining case when $m+n=4,5$ in Theorem 1.3. Let $T_{k}^{n}:=\mathbb{R}^{n} /\left(2^{k} \mathbb{Z}\right)^{n}$ denote the quotient of $\mathbb{R}^{n}$ for $k=0,1,2, \ldots$ Let $\left(\mathbb{R}^{n}, g_{\mathbb{E}}\right) \rightarrow\left(T_{k}^{n}, h_{k}\right)$ also denote the natural normal infinite Riemannian covering and

$$
P_{k}:\left(M^{m} \times \mathbb{R}^{n}, \bar{g}\right) \longrightarrow\left(M^{m} \times T_{k}^{n}, \bar{g}_{k}\right)
$$

the induced normal Riemannian covering, where $\bar{g}_{k}:=g+h_{k}$. Here note that each natural map

$$
\left(M^{m} \times T_{k+1}^{n}, \bar{g}_{k+1}\right) \longrightarrow\left(M^{m} \times T_{k}^{n}, \bar{g}_{k}\right)
$$

is also a finite Riemannian covering. Take any point $q \in M^{m}$ and fix it. Set $p=(q, 0) \in M^{m} \times \mathbb{R}^{n}$ and $p_{k}:=P_{k}(p) \in M^{m} \times T_{k}^{n}$. Then, for each $k$, there exists a unique normalized positive Green's function $G_{k}$ (with pole at $p_{k}$ ) for the conformal Laplacian $L_{\bar{g}_{k}}$ on $M^{m} \times T_{k}^{n}$, that is, $G_{k} \in C_{+}^{\infty}\left(\left(M^{m} \times T_{k}^{n}\right)-\right.$ $\left.\left\{p_{k}\right\}\right)$ with

$$
L_{\bar{g}_{k}} G_{k}=c_{m+n} \delta_{p_{k}} \text { on } M^{m} \times T_{k}^{n} \quad \text { and } \quad \lim _{x \rightarrow p_{k}} G_{k}(x)\left(\operatorname{dist}\left(p_{k}, x\right)\right)^{m+n-2}=1 .
$$

Here, $c_{m+n}>0$ and $\delta_{p_{k}}$ stand, respectively, for a specific universal constant and the Dirac $\delta$-function at $p_{k}$. The condition $\mathbf{s}_{g}>0$ implies that the first eigenvalue $\lambda\left(L_{\bar{g}_{0}}\right)>0$ on $M^{m} \times T_{0}^{n}$. By the condition $\lambda\left(L_{\bar{g}_{0}}\right)>0$, there exists a unique normalized minimal positive Green's function $G$ for $\mathrm{L}_{\bar{g}}$ with pole at $p \in M^{m} \times \mathbb{R}^{n}$. Moreover, there exist positive constants $a, b, K$ with $a<b, K \geq 1$ such that

$$
K^{-1} e^{-b|z|} \leq G(x) \leq K e^{-a|z|} \quad \text { for } \quad x=(y, z) \in M^{m} \times\left\{z \in \mathbb{R}^{n}|| z \mid \geq 1\right\} .
$$

See, $[2$, Section $6 ; 25]$ for details. 
Let us consider the family of the scalar-flat, asymptotically flat manifolds

$$
\left(X_{k}, \bar{g}_{k, \mathrm{AF}}\right):=\left(\left(M^{m} \times T_{k}^{n}\right)-\left\{p_{k}\right\}, G_{k}^{4 /(m+n-2)} \bar{g}_{k}\right) \quad \text { for } \quad k=0,1,2, \ldots
$$

and the one

$$
\left(X, \bar{g}_{\mathrm{AF}}\right):=\left(\left(M^{m} \times \mathbb{R}^{n}\right)-\{p\}, G^{4 /(m+n-2)} \bar{g}\right)
$$

with a singularity arising from the end of $M^{m} \times \mathbb{R}^{n}$. We denote the mass of $\left(X, \bar{g}_{\mathrm{AF}}\right)$ (resp. $\left.\left(X_{k}, \bar{g}_{k, \mathrm{AF}}\right)\right)$ by $\mathfrak{m}\left(\bar{g}_{\mathrm{AF}}\right)$ (resp., $\left.\mathfrak{m}\left(\bar{g}_{k, \mathrm{AF}}\right)\right)$. Then, an argument similar to the proof of [2, Theorem 6.13] implies

$$
\lim _{k \rightarrow \infty} \mathfrak{m}\left(\bar{g}_{k, \mathrm{AF}}\right)=\mathfrak{m}\left(\bar{g}_{\mathrm{AF}}\right)
$$

Hence, from the positive mass theorem [22-24], we obtain the following.

Lemma 4.3. Let $\left(X, \bar{g}_{\mathrm{AF}}\right)$ be the scalar-flat, asymptotically flat manifolds with a singularity and $\operatorname{dim} X=4,5$, defined as above. Then, $\mathfrak{m}\left(\bar{g}_{\mathrm{AF}}\right) \geq 0$.

Now we can complete the proof of Theorem 1.3.

Proof of Theorem 1.3. From Lemma 4.2, we consider the case when $m+$ $n=4,5$. We will prove that

$$
\mathfrak{m}\left(\bar{g}_{\mathrm{AF}}\right)>0
$$

Then, by an argument similar to the proofs of $[21$, Theorem $1 ; 26$, Chapter 5 , Theorem 4.1], the positivity of the mass $\mathfrak{m}\left(\bar{g}_{\mathrm{AF}}\right)>0$ implies the desired assertion

$$
Y\left(M^{m} \times \mathbb{R}^{n},[\bar{g}]\right)<\mathbf{Y}_{m+n} .
$$

Choose any large constant $L_{0}>0$ and fix it. Set

$$
X_{0}:=\left(M^{m} \times\left\{z \in \mathbb{R}^{n}|| z \mid \leq L_{0}\right\}\right)-\{p\} \subset X .
$$

Then, there exist harmonic coordinates near infinity $x=\left(x^{1}, \ldots, x^{m+n}\right)$ on $\left(X_{0}, \bar{g}_{\mathrm{AF}}\right)[5]$ (cf. [2, Lemma 6.17]). Namely, $\left(x^{i}\right)$ are smooth functions on $X_{0}$ satisfying

$$
\Delta_{\bar{g}_{\mathrm{AF}}} x^{i}=0 \text { on } X_{0}, \quad \frac{\partial x^{i}}{\partial \nu}=0 \text { on } \partial X_{0}
$$

and which give asymptotically flat coordinates near infinity of $\left(X_{0}, \bar{g}_{\mathrm{AF}}\right)$. Here, $\nu$ is the outward unit normal vector field normal to $\partial X_{0}$. 
Now we suppose that $\mathfrak{m}\left(\bar{g}_{\mathrm{AF}}\right)=0$. Note that, under this assumption, $\bar{g}_{\mathrm{AF}}$ is Ricci-flat on $X_{0}$. See [1, Lemma 3.1] for details (cf. [2, Proposition 6.14], [21, Lemma 3]). We now apply the Bochner technique to complete the proof. The harmonicity of $\left(x^{i}\right)$ implies that $\left\{d x^{i}\right\}$ are harmonic 1-forms on $\left(X_{0}, \bar{g}_{\mathrm{AF}}\right)$. From the Bochner formula for 1-forms $\left\{d x^{i}\right\}$ combined with the conditions that $\frac{\partial x^{i}}{\partial \nu}=0$ on $\partial X_{0}$ and $\operatorname{Ric}_{\bar{g}_{\mathrm{AF}}}=0$ on $X$, we have that (cf. [5, Theorem 4.4], [15, Proposition 10.2])

$$
\mathfrak{m}\left(\bar{g}_{\mathrm{AF}}\right)=\frac{1}{\operatorname{Vol}\left(S^{m+n-1}(1)\right)} \sum_{i=1}^{m+n} \int_{X_{0}}\left|\nabla d x^{i}\right|^{2} d \mu_{\bar{g}_{\mathrm{AF}}} .
$$

Then, by applying the mass zero condition $\mathfrak{m}\left(\bar{g}_{\mathrm{AF}}\right)=0$ in the above, we obtain that the 1 -forms $\left\{d x^{i}\right\}$ are parallel on $X_{0}$ with respect to $\bar{g}_{\mathrm{AF}}$. Since the coframe $\left\{d x^{i}\right\}$ is orthonormal at infinity, then $\left\{d x^{i}\right\}$ is a parallel orthonormal coframe everywhere on $\left(X_{0}, \bar{g}_{\mathrm{AF}}\right)$. This implies that the map $x=\left(x^{1}, \ldots, x^{m+n}\right):\left(X_{0}, \bar{g}_{\mathrm{AF}}\right) \rightarrow\left(\mathbb{R}^{m+n}, g_{\mathbb{E}}\right)$ is a local isometry, and hence $\bar{g}$ is locally conformally flat on $X_{0}$. This gives a contradiction to Lemma 4.1. Therefore, $\mathfrak{m}\left(\bar{g}_{\mathrm{AF}}\right)>0$. This completes the proof.

\section{Gagliardo-Nirenberg inequalities and Yamabe constants}

In this section, we estimate the behavior of arbitrary $N$-Yamabe constants in terms of the best constants in the Gagliardo-Nirenberg interpolation inequalities.

Let us define the $(m, n)$-Gagliardo-Nirenberg functional as

$$
\begin{aligned}
L(f)=L_{m, n}(f):= & \frac{\|\nabla f\|_{2}^{2 n /(m+n)}\|f\|_{2}^{2 m /(m+n)}}{\|f\|_{p_{(m+n)}}^{2}} \\
& \text { for } f \in W^{1,2}\left(\mathbb{R}^{n}\right) \text { with } f \neq 0 .
\end{aligned}
$$

Remark 5.1. The map $L$ is invariant under two operations. First, if $c$ is any non-zero constant then $L(c f)=L(f)$. Second, if $\lambda>0$ is a constant and $f_{\lambda}(x)=f(\lambda x)$ then

$$
\begin{aligned}
\left\|f_{\lambda}\right\|_{2}^{2 m /(m+n)} & =\lambda^{-m n /(m+n)}\|f\|_{2}^{2 m /(m+n)} \\
\left\|f_{\lambda}\right\|_{p_{m+n}}^{2} & =\lambda^{-2 n /\left(p_{n+m}\right)}\|f\|_{p_{m+n}}^{2}
\end{aligned}
$$

and

$$
\left\|\nabla f_{\lambda}\right\|_{2}^{2 n /(m+n)}=\lambda^{(2-n) n /(m+n)}\|\nabla f\|_{2}^{2 n /(m+n)} .
$$

Therefore, $L\left(f_{\lambda}\right)=L(f)$. 
Let us recall the following definition from the introduction.

Definition 5.2. The $(m, n)$-Gagliardo-Nirenberg constant is given by

$$
\sigma_{m, n}:=\left(\inf _{\substack{f \in W^{1,2}\left(\mathbb{R}^{n}\right) \\ f \neq 0}} L_{m, n}(f)\right)^{-1}
$$

Remark 5.3. The constant $\sigma_{m, n}$ has already been studied in the literature. It is the best constant for the classical interpolation inequality due to Gagliardo [8, 9] and Nirenberg [17] that says that $L_{m, n}$ is bounded away from zero. In [28], it is shown that $\sigma_{m, n}$ is closely related to the global existence of the nonlinear Schrödinger equation. The author showed also that $\sigma_{m, n}$ is always attained by a positive function $\psi \in W^{1,2}\left(\mathbb{R}^{n}\right) \cap C^{\infty}\left(\mathbb{R}^{n}\right)$, called a ground-state solution, that should then satisfy the corresponding EulerLagrange equation

$$
\Delta u-u+u^{q}=0, \quad q=\frac{m+n+2}{m+n-2}
$$

(the Euler-Lagrange equation for $L$ of course involves coefficients depending on $\|f\|_{2},\|f\|_{p}$ and $\|\nabla f\|_{2}$ : one can use the previous remark to normalize the equation as above). For any function $f \in W^{1,2}\left(\mathbb{R}^{n}\right) \cap C^{\infty}\left(\mathbb{R}^{n}\right)$, one can consider the spherical symmetrization $f^{*}$ of $f$, that is, the radial function $f^{*}$ satisfying $\operatorname{Vol}_{g_{\mathbb{E}}}\left\{f^{*}>t\right\}=\operatorname{Vol}_{g_{\mathbb{E}}}\{f>t\}$ for any positive $t$. It is a classical result that $L\left(f^{*}\right)<L(f)$ if $f^{*} \not \equiv f$. It follows that $\psi$ should be radial and decreasing. Finally, in [13], the uniqueness of the positive radial solution of (5.1) is proved under the assumption that it vanishes at infinity. The key point to our purposes is that these facts give a simple procedure to compute numerically all Gagliardo-Nirenberg constants. We will continue this discussion in Section 6.

We now relate the Gagliardo-Nirenberg constants with the $N$-Yamabe constant of limiting products.

Proof of Theorem 1.4. First fix a function $f \in C_{c}^{\infty}\left(\mathbb{R}^{n}\right)$ and consider the family of functions $f_{\lambda}$ as in Remark 5.1. Let us consider the map

$$
\lambda \longmapsto F(\lambda):=\frac{\int_{\mathbb{R}^{n}}\left(a_{m+n}\left|\nabla f_{\lambda}\right|_{g_{\mathbb{E}}}^{2}+\mathbf{s}_{g} f_{\lambda}^{2}\right) d \mu_{g_{\mathbb{E}}}}{\left\|f_{\lambda}\right\|_{p_{m+n}}^{2}} .
$$


If we set

$$
A:=\frac{\int_{\mathbb{R}^{n}} a_{m+n}|\nabla f|_{g_{\mathbb{E}}}^{2} d \mu_{g_{\mathbb{E}}}}{\|f\|_{p_{m+n}}^{2}}
$$

and

$$
B:=\frac{\int_{\mathbb{R}^{n}} \mathbf{s}_{g} f^{2} d \mu_{g_{\mathbb{E}}}}{\|f\|_{p_{m+n}}^{2}},
$$

then Remark 5.1 implies that $F(\lambda)=\lambda^{2 m /(m+n)} A+\lambda^{-2 n /(m+n)} B$. We see that this map achieves its minimum at $\lambda_{0}=\sqrt{\frac{n B}{m A}}$ and that this minimum is

$$
\begin{aligned}
F\left(\lambda_{0}\right) & =A^{n /(m+n)} B^{m /(m+n)} m^{-m /(m+n)} n^{-n /(m+n)}(m+n) \\
& =\mathbf{s}_{g}^{m /(m+n)} C(m, n) L(f) .
\end{aligned}
$$

Recall that $L(f)=L\left(f_{\lambda}\right)$. Therefore,

$$
\begin{aligned}
\lim _{r \nearrow \infty} Y_{N^{n}}\left(M^{m} \times N^{n}, g+r h\right) & =Y_{\mathbb{R}^{n}}\left(M^{m} \times \mathbb{R}^{n}, g+g_{\mathbb{E}}\right) \\
& =\inf _{\substack{f \in C_{c}^{\infty}\left(\mathbb{R}^{n}\right) \\
f \neq 0}} \frac{\int_{\mathbb{R}^{n}}\left(a_{m+n}|\nabla f|_{g_{\mathbb{E}}}^{2}+\mathbf{s}_{g} f^{2}\right) d \mu_{g_{\mathbb{E}}}}{\|f\|_{p_{m+n}}^{2}} \\
& =\inf _{\substack{f \in C_{c}^{\infty}\left(\mathbb{R}^{n}\right) \\
f \neq 0}} \mathbf{s}_{g}^{m /(m+n)} C(m, n) L(f) \\
& =\frac{\mathbf{s}_{g}^{m /(m+n)} C(m, n)}{\sigma_{m, n}} .
\end{aligned}
$$

\section{Numerical computations}

We now describe a procedure to determine all Gagliardo-Nirenberg constants $\sigma_{m, n}$ numerically, and how we obtained our table in the introduction.

Consider the solution $h=h_{\alpha}: \mathbb{R}_{\geq 0} \rightarrow \mathbb{R}$ of the initial value problem

$$
\begin{aligned}
& h^{\prime \prime}(t)+\frac{n-1}{t} h^{\prime}(t)-h(t)+h(t)^{(m+n+2) /(m+n-2)}=0, \\
& h(0)=\alpha>0, \quad h^{\prime}(0)=0 .
\end{aligned}
$$

This equation corresponds to the critical points of the $(m, n)$-GagliardoNirenberg functional $L_{m, n}$ (see Remarks 5.1 and 5.3), when restricted to radial functions $u(x)=h(|x|)$, which is enough to consider because of symmetrization. By the existence result in [28] and its uniqueness finally proved 


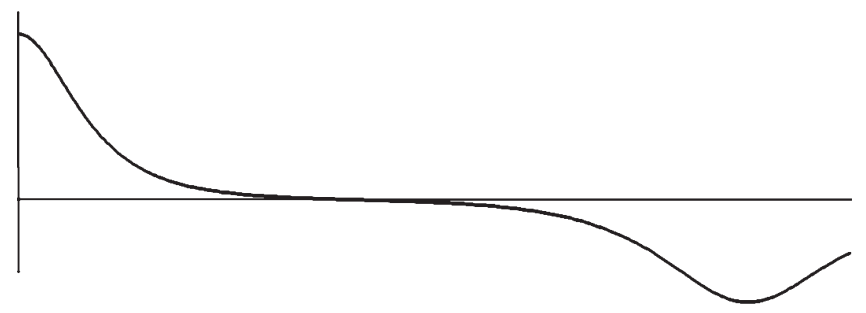

Figure 1: $h_{\alpha}$ for $\alpha>\alpha_{0}$.

in [13], there exists only one value $\alpha=\alpha_{0}=\alpha_{0}(m, n)$ that gives a positive solution $h_{\alpha_{0}}$ that vanishes at infinity, called the ground state. To find $\alpha_{0}$ numerically, we use [13] where it is shown that, for values $\alpha>\alpha_{0}$, the solution $h_{\alpha}$ vanishes exactly once and then oscillates about -1 (figure 1), while, for values $\alpha<\alpha_{0}$, it is positive and oscillatory about the value 1 (figure 2).

A key point is the uniqueness of the solution of (6.1) when the initial value condition $h(0)=\alpha$ is replaced by the boundary condition $h\left(t_{0}\right)=$ 0 , for $t_{0} \in(0,+\infty]$, and the fact that this solution has $h^{\prime}<0$ in $\left(0, t_{0}\right]$; see [13, Lemmas 9 and 11 and the main Theorem page 265]. Finally, by [28], we have that $\sigma_{m, n}^{-1}=L_{m, n}\left(h_{\alpha_{0}}\right)$. For example, we can then compute $\alpha_{0}=\alpha_{0}(2,2) \approx 2.2062$ for the ground state initial value, and hence $\sigma_{2,2}^{-1}=L_{2,2}\left(h_{\alpha_{0}}\right) \approx 2.41877$. In fact, figure 1 corresponds to $m=n=2$ and $\alpha=2.208$ and figure 2 to $\alpha=2.205$.

Of course, if one wants to avoid the numerical computation, one could give estimates for $\sigma_{m, n}$ by carefully choosing functions. For instance, we can show that $\sigma_{2,2}^{-1}<2.427458<\sqrt{2 \pi}$ by considering the function $h: \mathbb{R}_{\geq 0} \rightarrow \mathbb{R}$ that linearly interpolates the following data: $h(0)=1, h(0.1)=0.9904132$, $h(0.2)=0.9626, h(0.3)=0.91917, h(0.7)=0.66607, h(0.9)=0.5378, h(1.15)$ $=0.4023, h(1.3)=0.34, h(1.5)=0.2634, h(1.85)=0.17201, h(2.2)=0.11288$, $h(2.6)=0.07031, h(3)=0.04416, h(3.5)=0.02493, h(3.9)=0.016, h(4.3)=$ $0.01016, \quad h(5)=0.0047, h(6)=0.00158, \quad h(7)=0.00054, \quad h(8)=0.00019$,

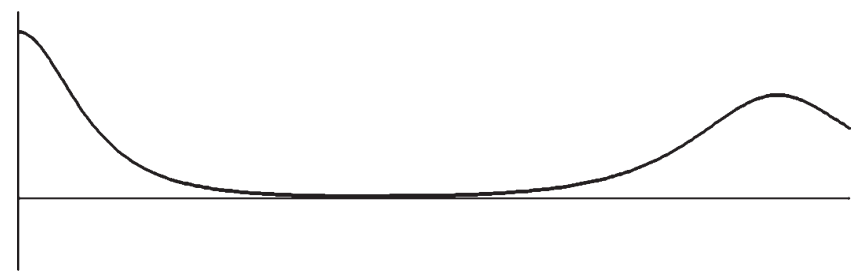

Figure 2: $h_{\alpha}$ for $\alpha<\alpha_{0}$. 
$h(9)=0.00006$, and $h(t)=0$, for $t \geq 10$. Now, define the radial function $f: \mathbb{R}^{2} \rightarrow \mathbb{R}$ given by $f(x)=h(|x|)$. A straightforward computation gives that $\sigma_{2,2}^{-1} \leq L_{2,2}(f)<2.427458$. And then

$$
Y_{S^{2}}^{\infty}\left(S^{2} \times S^{2}\right)<2.427458(8 \sqrt{3 \pi})<8 \sqrt{6} \pi=\mathbf{Y}_{4}
$$

\section{Acknowledgments}

The authors would like to thank Professors Manuel del Pino and Jean Dolbeault for valuable observations on the Gagliardo-Niremberg inequalities and Fernando Coda for numerous conversations on the subject. The first author would like to express his gratitude to Professors Boris Botvinnik and Osamu Kobayashi for many helpful discussions. The third author would like to express his gratitude to IMPA where this work was carried on. K.A. is supported in part by the Grants-in-Aid for Scientific Research (C), Japan Society for the Promotion of Science, No. 16540059. L.A.F. is partially supported by CNPq-FAPERJ, Brazil. J.P. is supported by grant 46274-E of CONACYT and CAPES-Brazil.

\section{References}

[1] K. Akutagawa, Aubin's lemma for the Yamabe constants of infinite coverings and a positive mass theorem, Preprint, 2007.

[2] K. Akutagawa, B. Botvinnik, Yamabe metrics on cylindrical manifolds, Geom. Funct. Anal. 13 (2003), 259-333.

[3] T. Aubin, Equations différentielles non-linéaires et problème de Yamabe concernant la courbure scalaire, J. Math. Pures Appl. 55 (1976), 269-296.

[4] T. Aubin, Some Nonlinear Problems in Riemannian Geometry, Springer Monographs in Mathematics, Springer-Verlag, New York, 1998.

[5] R. Bartnik, The mass of an asymptotically flat manifold, Comm. Pure. Appl. Math. 39 (1986), 661-693.

[6] B. Botvinnik, J. Rosenberg, The Yamabe invariant of non-simply connected manifolds, J. Differential Geom. 62 (2002), 175-208. 
[7] L. Caffarelli, B. Gidas, J. Spruck, Asymptotic symmetry and local behavior of semilinear elliptic equations with critical Sobolev growth, Comm. Pure Appl. Math. 42 (1989), 271-297.

[8] E. Gagliardo, Proprieta di alcune classi di funzioni in piu varibili, Ricerche di Math. 7 (1958), 102-137.

[9] E. Gagliardo, Ulteriori proprieta di alcune classi di funzioni in piu variabili, Ricerche di Math. 8 (1959), 24-51.

[10] M. Gursky, C. LeBrun, Yamabe invariants and Spin ${ }^{c}$-structures, Geom. Funct. Anal. 8 (1998), 965-977.

[11] O. Kobayashi, On large scalar curvature, Research Report 11, Department of Mathematics Keio University, 1985.

[12] O. Kobayashi, Scalar curvature of a metric with unit volume, Math. Ann. 279 (1987), 253-265.

[13] M. Kwong, Uniqueness of positive solutions of $\Delta u-u+u^{p}=0$ in $R^{n}$, Arch. Rational Mech. Anal. 105 (1989), 243-266.

[14] C. LeBrun, Yamabe constants and the perturbed Seiberg-Witten equations, Comm. Anal. Geom. 5 (1997), 535-553.

[15] J. Lee, T. Parker, The Yamabe problem, Bull. Amer. Math. Soc. (N.S.) 17 (1987), 37-91.

[16] H. Levine, An estimate for the best constant in a Sobolev inequality involving three integral norms, Ann. Mat. Pura Appl. 124 (1980), 181-197.

[17] L. Nirenberg, On elliptic partial differential equations, Ann. Scuola Norm. Sup. Pisa 13 (1959), 116-162.

[18] J. Petean, G. Yun, Surgery and the Yamabe invariant, Geom. Funct. Anal. 9 (1999), 1189-1199.

[19] J. Petean, Computations of the Yamabe invariant, Math. Res. Lett. 5 (1998), 703-709.

[20] J. Petean, The Yamabe invariant of simply connected manifolds, J. Reine Angew. Math. 523 (2000), 225-231.

[21] R. Schoen, Conformal deformation of a Riemannian metric to constant scalar curvature, J. Differential Geom. 20 (1984), 479-495. 
[22] R. Schoen, Variational theory for the total scalar curvature functional for Riemannian metrics and related topics, Lectures Notes in Mathematics, 1365, Springer-Verlag, Berlin, 1989, 120-154.

[23] R. Schoen, S.-T. Yau, On the proof of the positive mass conjecture in general relativity, Comm. Math. Phys. 65 (1979), 45-76.

[24] R. Schoen, S.-T. Yau, Proof of the positive mass theorem. II, Comm. Math. Phys. 79 (1981), 231-260.

[25] R. Schoen, S.-T. Yau, Conformally flat manifolds, Kleinian groups and scalar curvature, Invent. Math. 92 (1988), 47-71.

[26] R. Schoen, S.-T. Yau, Lectures on Differential Geometry, Conferences Proceedings and Lecture Notes in Geometry and Topology I, International Press, Cambridge, MA, 1994.

[27] N. Trudinger, Remarks concerning the conformal deformation of Riemannian structures on compact manifolds, Ann. Scuola Norm. Sup. Pisa 22 (1968), 265-274.

[28] M. Weinstein, Nonlinear Shrödinger equations and sharp interpolation estimates, Comm. Math. Phys. 87 (1983), 567-576.

[29] H. Yamabe, On a deformation of Riemannian structures on compact manifolds, Osaka Math. J. 12, 21-37.

Department of Mathematics

Tokyo University of Science

NODA 278-8510

JAPAN

E-mail address: akutagawa_kazuo@ma.noda.tus.ac.jp

IMPA Est. Dona Castorina 110

22460-320 RIO DE JANEIRO

BRAZIL

E-mail address: luis@impa.br

CIMAT

A.P. 402

36000 Guanajuato

GTO

MÉXICO

E-mail address: jimmy@cimat.mx

ReCEIVED ApRIL 13, 2006 